\title{
Linear Intensity-Based Image Registration
}

\author{
Yasmin Mumtaz Ahmad ${ }^{1}$, Shahnorbanun Sahran², \\ Afzan Adam ${ }^{3}$ \\ Center for Artificial Intelligence Technology, \\ Faculty of Information Sciences and Technology, \\ Universiti Kebangsaan Malaysia,43600 Bangi, Malaysia
}

\author{
Syazarina Sharis Osman ${ }^{4}$ \\ Department of Radiology \\ Hospital Canselor Tuanku Mukhriz \\ Cheras, Universiti Kebangsaan Malaysia, \\ 56000 Kuala Lumpur, Malaysia
}

\begin{abstract}
The accurate detection and localization of lesion within the prostate could greatly benefit in the planning of surgery and radiation therapy. Although T2 Weighted Imaging (T2WI) Magnetic Resonance Imaging (MRI) provides an infinite amount of anatomical information, which ease and improve diagnosis and patient treatment, however, modality specific image artifacts, such as the occurrences of intensity inhomogeneity are still obvious and can adversely affect quantitative image analysis. Conventional high resolution T2WI has been restricted in this respect. On the contrary, Apparent Diffusion Coefficient (ADC) map has been seen as capable to tackle T2WI limitation when a functional assessment of the prostate capable to provide added value compared to T2WI alone. Likewise, it has been shown that diagnosis using ADC map combined with T2WI significantly outperforms T2WI alone. Therefore, to obtain high accuracy detection and localization, a combination of high-resolution anatomic and functional imaging is extremely important in clinical practice. This strategy relies on accurate intensity based image registration. However, registration of anatomical and functional MR imaging is really challenging due to missing correspondences and intensity inhomogeneity. To address this problem, this study researches the used of applying linear geometric transform to the corresponding point to accurately mapping the images for precise alignment and accurate detection. Transformation type is crucial for the success of image registration. The selection of transformation type is influenced by the type and severity of the geometric differences between corresponding images, the accuracy of the control point between images, its density and organization of the control points. A transformation type is selected to reflect geometric differences between two images in image registration. Often, the selection of the suitable transformation type for image registration is undeniably challenging. To make this selection as effective as possible, an experimental mechanism has to be carried out to determine its suitability. These transformations types are Affine, similarity, rigid and translation. Additionally, intensity based image registration is implemented to optimize the similarity metric mean square error through regular step gradient descent optimizer. Accuracies evaluation for each transformation type has been carried out through mean square error (MSE) and peak signal noise ratio (PSNR). The results have been presented in a chart form together with a comparison table.
\end{abstract}

Keywords-Lineargeo metric transformation; image registration; point correspondence

\section{INTRODUCTION}

The treatment of prostate cancer could greatly benefit from discovering imaging markers in MRI images that accurately predict existence lesion. Although MRI provide a massive amount of anatomical and functional information, which ease expert's daily task and improve diagnosis and patient treatment, However, modality specific image artefacts, such as the phenomena of intensity inhomogeneity in MRI, are still obvious and can adversely affect quantitative image analysis[1]. Image artefacts exist due to variability between patients during the MRI examinations even using the same scanner, protocol or sequence parameters [2]. The researcher in [3] and other researchers have emphasized the need to develop a system that can extract quantitative data in a more accurate and automated fashion. One possible approach for discovering these markers is to first align the anatomical and functional MR of a patient and then to analyse the imaging characteristics of suspicious lesion. This strategy relies on accurate registration. However, registration of anatomical and functional MR imaging is very challenging due to missing correspondences and intensity inhomogeneity between the MR imaging. To address this problem, this study researches the used of applying linear geometric transform to corresponding point to accurately maps the images for precise alignment and better observation. The process of mapping points from a reference image to the corresponding points in the target image is identified as an intensity based image registration[4]. Image registration comprises of four major components, which are a feature selection, a transformation model, a similarity metric and an optimization method. This paper examines the second step in image registration that is, transformation that is used to map corresponding points in the images. The type of transformation selected is vary depending on the type and density of the geometric differences between the images, the accuracy and organization of the matching control point [4].Although defining transformation type selection are essential, this paper will not cover the selection transformation criteria. Many authors have discussed method for transformation type selection. This paper presents the analysis for image transformation model, which is the second step in image registration. The linear geometric transformation will be investigated due to the fact that it is adequate to solve medical image registration acquired from same sensors [4]. This paper will examine the used of linear transformations for aligning two dimension (2D) Magnetic Resonance images (MRI) acquired from the same sources. The images are labelled as reference image for T2 Weighted Imaging (T2WI) and target for Apparent Diffusion Coefficient (ADC) image. These labelled will be consistently used in the entire paper. Both images acquired from axial plane as shown in Fig. 3a and $3 \mathrm{~b}$. 


\section{A. Prostate Cancer}

The Prostate is divided into three glandular zones : the peripheral zone contains 70 percent of the glandular tissue and accounts about 70 percent of prostate cancer[5]. The transition zone contains 5 percent of the gland tissues and accounts for 25 percent of prostate cancer[5] and the central zone contains 20 percent of glandular tissue and account most fewer percent of prostate cancer[5].Accurate early detection within peripheral zone and transitional zone is crucial as both zones are associated with favourable pathologic features and better recurrence free survival[5]. At present, clinical procedure for prostate cancer diagnosis is trans-rectal ultrasound and systematic biopsy.

\section{B. MR Image}

Conventional MRI of the prostate combines anatomic images T2WI and functional information obtained from ADC. This combination is identified as multi parametric-MRI(MPMRI). This section clarifies the sequence used by radiologists in their daily diagnosis task. These sequences are T2WI and ADC.

1) T2-weighted imaging (T2WI): The first and most important step in an MP-MRI protocol used to perform diagnosis as it is well suited to render zonal anatomy of the prostate[6].Peripheral zone and central gland tissues are well observable in these image[7]. On this sequence, prostate cancer generally demonstrates low signal intensity (SI) contrast, to the high SI on the normal peripheral zone[8]. A sample of this image can be seen in Fig. 3a with delineated tumour. Since, T2WI is most important sequence to locate prostate cancer, therefore, it is the most frequently used sequence in computer aided detection system.

2) Apparent diffusion coefficient (ADC): Diffusion weighted imaging (DWI) measures the diffusion of water molecules within different tissues[9]. Normal prostate gland tissue has a higher water diffusion rate than cancer tissue[9].DWI is inherently T2WI but, unlike conventional T2WI, prostate cancer usually demonstrates increased SI on DWI effecting the lesion difficult to visualize within the normal high signal peripheral zone[9].To minimize the effect of this T2WI sine through effect that may result in the depiction of a false positive high intensity lesion[10], the ADC is calculated[8], which is calculated based on diffusionweighted imaging(DWI) using various b values, is useful for differentiate existence lesion either benign or malignant [11].Furthermore, Prostate lesion visible as a high signal region on DWI but as low signal region on ADC map. Fig. 3b shows an ADC map with delineated lesion as low signal intensity (arrow).

\section{Types of Linear Transformation}

1) Affine: A common affine transformation from $2 \mathrm{D}$ to $2 \mathrm{D}$ as in below equation 1 derived from [12] which requires six parameters and computed from only three matching pairs of points [12]

[[xi ; yi ]; [Xi; Yi ]]i=1;3]
$\left[\begin{array}{l}\mathrm{X} \\ \mathrm{Y} \\ 1\end{array}\right] \square\left[\begin{array}{ccc}\mathrm{a} 11 & \mathrm{a} 12 & \mathrm{a} 13 \\ \mathrm{a} 21 & \mathrm{a} 22 & \mathrm{a} 23 \\ 0 & 0 & 1\end{array}\right] \square\left[\begin{array}{l}\mathrm{X} \\ \mathrm{y} \\ 1\end{array}\right]$

2) Similarity: The similarity transform represent global translation, rotation and scaling dissimilarities among reference and target images [13] and only need two point control. Equations 3 derived from [13] represent similarity transforms

$\mathrm{X}=\mathrm{xs} \cos \theta-\mathrm{ys} \sin \theta+\mathrm{h}$

$\mathrm{Y}=\mathrm{xs} \sin \theta+\mathrm{ys} \cos \theta+\mathrm{k}$

Where

$\mathrm{s}, \theta$, and $(\mathrm{h}, \mathrm{k})$ are scaling, rotational, and translation dissimilarities between the images. These parameters could be easily identified if the images correspondence control point coordinates are known. The rotational dissimilarity between the images is determined from the angle between the lines connecting the two points in the images. The scaling differences between the images are determined from the ratio of distances between the points in the images.

3) Rigid: A rigid transformation is described with translations and rotations. It has only three parameters as represented in equations 5.

$\mathrm{T}$ linear $(\mathrm{x})=\mathrm{Rx}+\mathrm{t}$

Where

$R$ is a rotation matrix and $t$ is a translation vector.

4) Translation: Two translation parameters required for 2dimension transformation as represents in equations 6 . Translated by the vector [Tx, Ty] is

$f_{T}(x, y)=f(x-T x, y-T y)$

Where

$\mathrm{T}_{\mathrm{x}}$ is the horizontal translation and $\mathrm{T}_{\mathrm{y}}$ is the vertical translation.

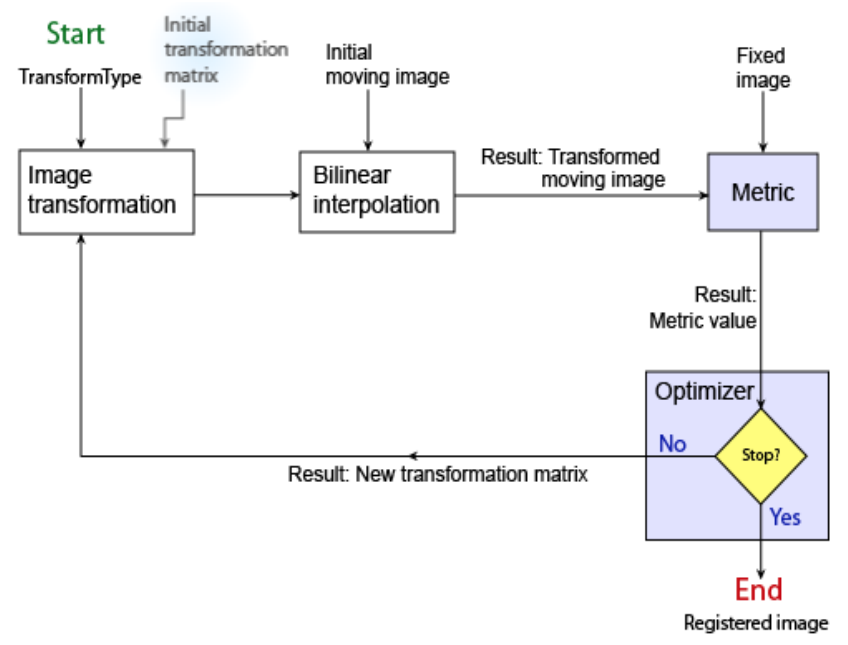

Fig. 1. Iterative Process of Intensity based Image Registration. Source from [16]. 


\section{THE MATERIAL AND METHOD}

Matlab has been adopted to evaluate the transformed images accuracy as it represents images in a matrices form [14]. An image is a M x N array of elements. Each element in the array is known as an intensity value. Intensity based image registration is an iterative process. To summarize the registration process, Fig.1 illustrates the iterative process of image registration. The ultimate goal of image registration is to search iteratively for a geometrical transformation that will minimizes the target image similarity metric until termination criteria is met [15]. For this iterative processes success, it requires a pair of images, a similarity metric, an optimizer and a transformation models. As mentioned in previous section, since this research will be registering images from the same sensor i.e. MRI, linear geometric transformations have been chosen, as this transformation model are seemingly adequate with the problem needs to be solved. The similarity metric calculates the image correspondences for weighing the accuracy of image registration. The optimizer describes the method for minimizing the similarity metric and the transformation model will transform the target image as a reference image. MR Images Acquisition.

The MR dataset obtained by the 3 Tesla(T) MRI Siemens Verio contained T1-weighted imaging, T2-weighted imaging, DWI with Apparent Diffusion Coefficient(ADC) mapping and dynamic contrast enhanced imaging(DCE)[17].Each patient was scanned with the clinical standard MRI protocol. Multiple acquisition in three orthogonal planes (axial, coronal, sagittal) were performed using high resolution T2-weighted. ADC maps were obtained from the DWI sequences[8].The size of each sequences is differ according to resolution. The size of T2 weighted imaging is a $512 \times 512$. The radiologist manually segmented the whole prostate. In addition, the ethical protocol was obtained and approved by the internal ethics committee and informed consent was obtained from the patients.

\section{A. MR Image Analysis}

All acquired sequences were reviewed for each patient. Initially, The T2WI has to be reviewed for the area of hypo intensity in contrast to the high signal intensity of the normal peripheral zone as shows in Fig. 3a. While on the ADC map the criteria for lesion existence were low signal intensity as depicted in Fig.3b.

\section{B. Linear Geometric Transformation}

Transformation model is one of the main characteristics of the image registration in order to precisely align reference and target images. In this section the linear transformation type will be explained further. Linear transformation changes only the spatial property of images (horizontal, vertical up,down)and does not change the property of pixels i.e intensity, color, shape [18]. This research study particularly about points transformation between two dimensions' spaces.. A 2D point has two coordinates and frequently denoted as either a row vector $\mathrm{U}=[\mathrm{x}, \mathrm{y}]$ or column vector $\mathrm{U}=[\mathrm{x}, \mathrm{y}] \mathrm{t}[12]$. Therefore, the used of homogeneous coordinates for points is more convenient as the selected transformation model is linear geometric transformation.
Linear geometric transformations is defined as a mapping of any straight line to a straight line [19] . It is classified into four categories: Affine, Similarity, Rigid and Translation. The simplest linear transformation model is a horizontal or vertical shift. This shifting transformation is known as translation.[20]. Affine is the most complex linear transformation includes of Translation, rotation, scaling and shear. Similarity is a composition of translation, rotation and scaling. Rigid is a combination of translation and rotation. In the next section, the geometric transformations are further discussed in mathematical representation form. In section 3 a comparison result between these models were represents. Detail explanation of linear geometric transformation as in equation 7,8 and 9 adapted from [19] represents coordinates of $M$ pairs control points in two images of the same source.

$[[x j, y j] ;[X j, Y j]: j=1: M]$

where a transformation function $f(x, y)$ with elements $\mathrm{f}_{\mathrm{x}}(\mathrm{x}, \mathrm{y})$ and $\mathrm{f}_{\mathrm{y}}(\mathrm{x}, \mathrm{y})$ that fulfill

$[X j=f x(x j, y j)]$

$[Y j=f y(x j, y j), j=1, \ldots, M]$

Once the coordinates of a point $(x, y)$ in $f(x, y)$ is identified, then the coordinates of the matching points in the target image can be identified as well. Here, the $(\mathrm{x}, \mathrm{y})$ is referred as reference coordinates and $(\mathrm{X}, \mathrm{Y})$ as target image coordinates. The reference image is reserved basic while the target image transform according to reference image.

\section{Similarity Measure}

Similarity measure computes the quantity of similarity between intensity in two images [21] [22]. The choice of an image similarity metric depends on the modality of the images needs to be registered [22]. The similarity metric used in this research is Mean Square Error (MSE).This metric is commonly used with mono-modal intensity based problem, which is the reason adopted for this research as this research is also solving mono-modal intensity based problem. The MSE is a cumulative squared error between transformed and reference image [22]. The mathematical representation for measuring MSE as in equation 10 derived from [22].

$\mathrm{MSE}=1 / \mathrm{MN}^{*} \sum_{\mathrm{y}=1}^{\mathrm{M}} \sum_{\mathrm{x}=1}^{\mathrm{N}}\left[\mathrm{J}(\mathrm{x}, \mathrm{y})-\mathrm{J}^{\prime}(\mathrm{X}, \mathrm{Y})\right]^{2}$

Where $\mathrm{J}(\mathrm{x}, \mathrm{y})$ is a reference image, $\mathrm{J}$ ' $(\mathrm{X}, \mathrm{Y})$ is the target image and $\mathrm{M}, \mathrm{N}$ are the dimensions of the images. A lower value signifies lower similarity error and higher similarity between the images [11]

\section{Optimizer}

Regular Step Gradient Descent (RSGD) is applied as optimization method. This optimizer suits most for minimization problem in image registration [23].Hence, it is chosen to minimize the similarity measure between two images. It initially moved from initial trial point $\mathrm{X}^{1}$ moved along the steepest descent direction until the optimal point is found [23] as shown in Fig. 2. This optimizer will not terminate the iteration unless it reaches an optimal point. RSGD adopting hill climbing method begins with an opening estimate $\mathrm{X}^{\mathrm{k}}$ of the MSE. 


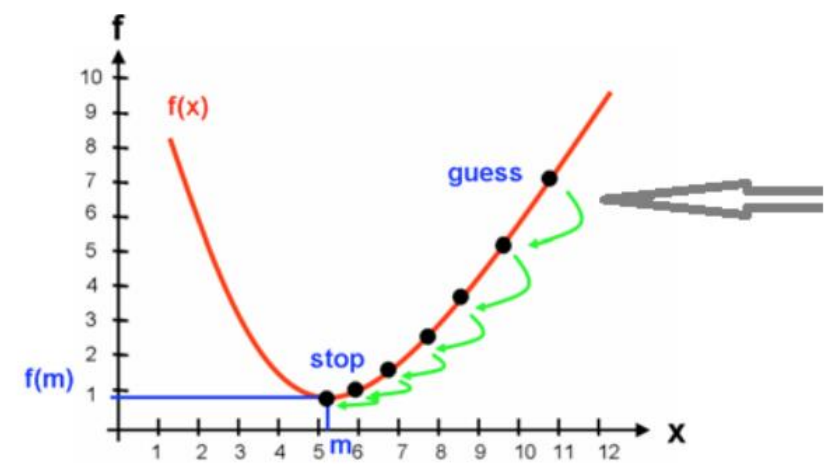

Fig. 2. Regular Step Gradient Descent Optimizer.Source from [12].

\section{RESULTS AND DISCUSSION}

In this section, the registrations of linear geometric transform between 2D images are presented. To register a target image $J^{\prime}$ to a reference image $J$, a six parameter transformation (parameterized by q1, q2, q3, q4, q5q, q6) has been used. A dot in the reference image that is denoted by $\mathrm{Xj}$ is compared with dot in the target image. As stated in previous section, the parameters $q 1-q 6$ are optimized by minimizing the mean square error between the images using Regular step gradient descent (RSGD).

\section{A. Medical Image Registration using Linear Geometrical Transformation}

The iteration started with the chosen 2D transformation, which is in this research a linear geometric transformation. The purpose of this transformation is to align precisely the target image ADC with the reference image T2WI. Both images were 8 bit but the width and height of both images are different; T2WI is $1080 \times 1080$ whereas ADC is $910 \times 1080$ as shown in Fig. 3a and $3 \mathrm{~b}$ respectively and acquired by same sources. Thus, it is necessary to resize the ADC equally with $\mathrm{T} 2 \mathrm{~W}$ before the alignment process begins. The ADC image were resizing with $\mathrm{Bi}$-cubic interpolation. Fig.3a (reference Image) and $3 b$ (target Image) are MR images of a patient has prostate tumour. Tumours are marked with red arrows in respective images. Intensity histograms of both MR images are attached besides respective MR image. These MR images are taken at the same time using the same source.

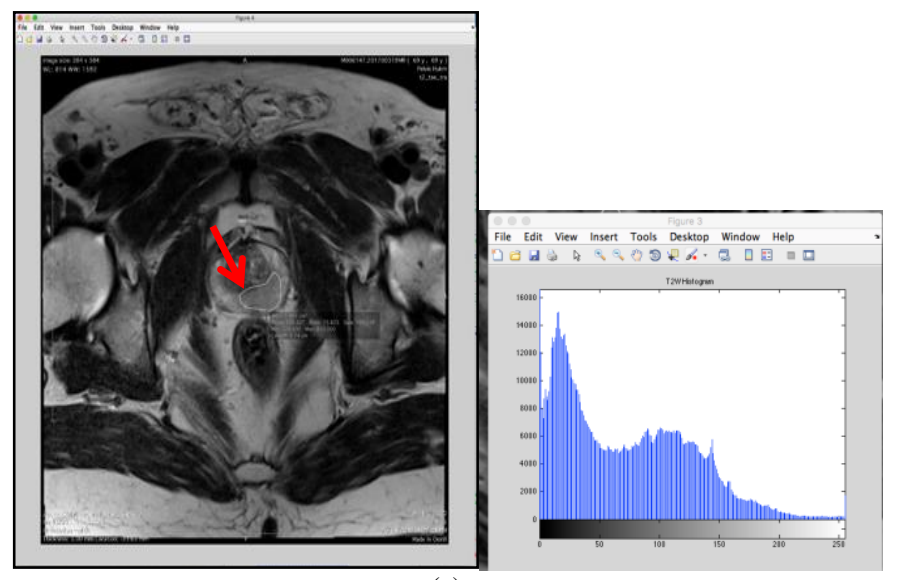

(a)

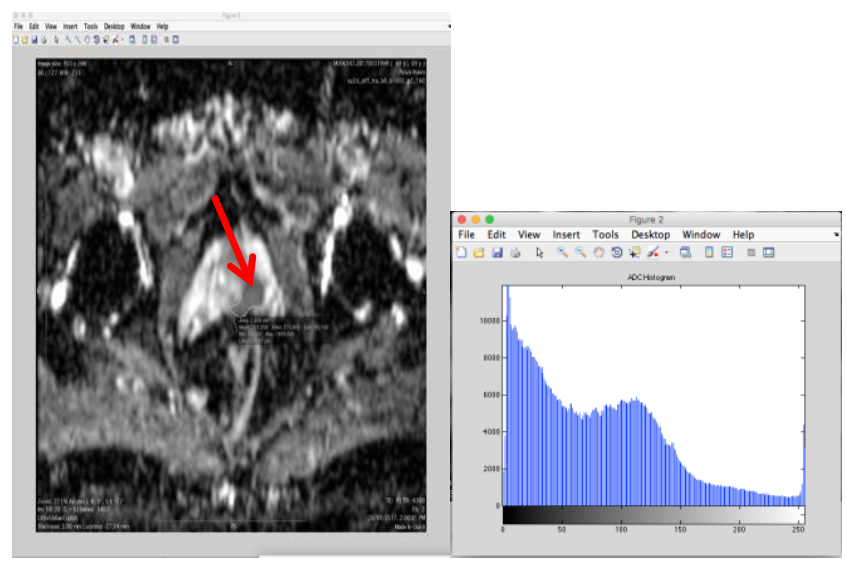

(b)

Fig. 3. (a) Axial Plane T2WI Shows an Ill Defined Hypointense Lesion (Arrow) at Peripheral Zone.also this Sequence Labelled as Reference Image (T2W). Attached besides, is a Histogram Intensity for T2WI., (b) Low SI (Arrow) on ADC Map.Attached besides is a Histogram Intensity for ADC Map.

Next, the metric which is Mean squares error has been applied to compares the value of transformed ADC to T2W.Mean square error (MSE), Sum square differences (SSD) and cross correlation (CC) are commonly used for image registration acquired from the same modality (Panda, et all, 2017). Thus, MSE is applied as due to less computation time and complexity. Finally, the optimizer checks for a stopping condition. The optimizer search the minimum value of the similarity metric before it is terminated when the similarity metric reach the stopping condition [23].In this paper, regular step gradient decent has been used as optimization method. This optimization method can be expressed as in equation 11 based on [23]

$\operatorname{minimize}_{x \in R}{ }^{n} f(x)$

Where, the objective function $f(x)$ is to locate the local minima of a transform using RSGD.

Fig. 4 shows the unregistered overlapping result of two MR images. Misregistration between two images is clearly visible as marked with red arrow. Expert has marked abnormality in both images manually. The purple is a reference image and the green is a target image.

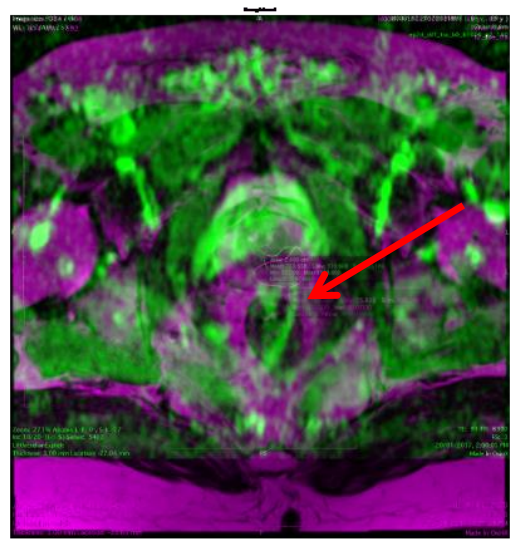

Fig. 4. Unregistered Overlapping of Reference and Target Images. 
Linear Transformation models: Affine, Similarity, Rigid and translation has been implemented and evaluated for monomodal prostate MR images. Fig. 5a-5h show the experimental results and represents histogram intensity of each linear geometrical transformation. The $\mathrm{x}$ and $\mathrm{y}$ axes in histogram represent intensity of the images. Fig. 5a shows an experimental registration result for affine transformation and generated histogram intensity. As seen here, rather than agreeing a homogenously spaced grid to select image blocks globally, affine transformation only adopt the locally spaced to select the image block. This is because Affine transformation originate from a combination of scaling, rotation, shearing and translation transformation [24].Hence, applying Affine transformation only useful when dealing with locally transformation[24].

Fig. 5c shows an experimental result for similarity transform and similarity intensity histogram. From this result, it can be seen that, similarity transformation is not suitable for registration of images acquired from same sources and images with global geometric differences. Fig. 5e shows an experimental result for rigid transformation and it intensity histogram. Fig. 5g shows an experimental result for translation transformation and it histogram intensity. It can be seen that, a rationally good accuracy was attained at and near the control points. This can be concluded that translation basis is reasonably good for registering images with global geometric differences which is uniformly spaced control points and images acquired from same modality. Table 1 illustrates evaluation summary of each linear transformation types. Mean, standard deviation, mean square error (MSE) and Peak signal noise ratio (PSNR) were applied to show the results.

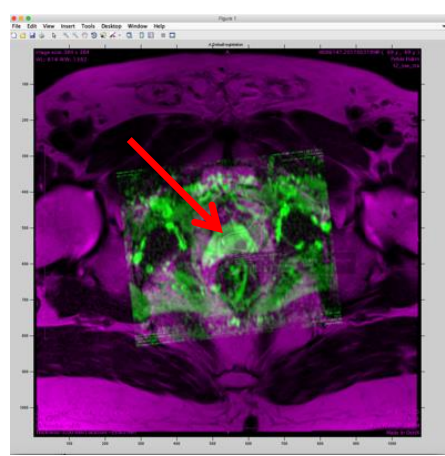

(a)

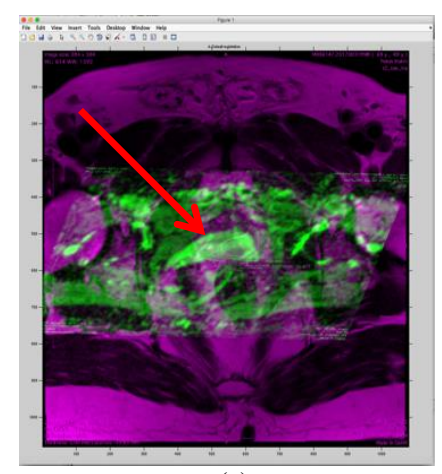

(c).

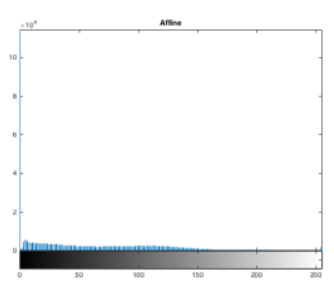

(b)

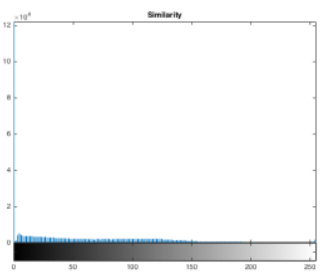

(d)

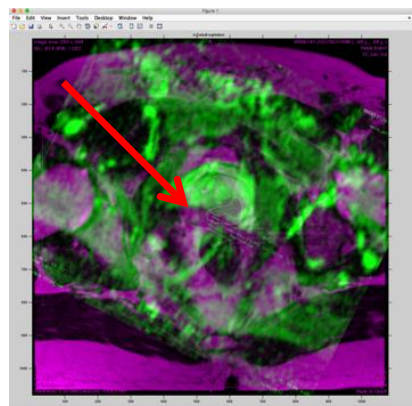

(e)

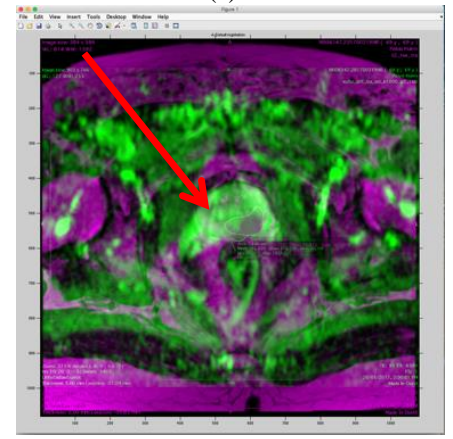

(g)

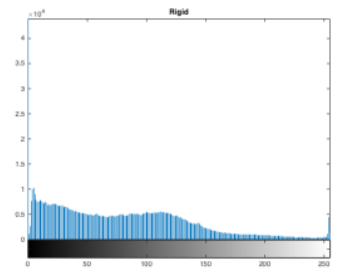

(f)

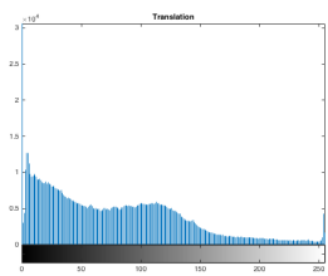

(h)
Fig. 5. (a) Affine Transformation., (b) Affine Intensity., (c) Similarity Transformation, (d) Similarity Intensity., (e) Rigid Transformation., (f) Rigid Intensity., (g) Translation Transformation. , (h)Translation Intensity.

\section{B. Quantitative Measures of Image Registration Accuracy}

To demonstrate the accuracy and robustness of each geometrical transformation model, it is necessary to evaluate registration accuracy. Measures techniques were used to evaluate the quality of the registration are mean, standard deviation, mean square error (MSE) and peak signal noise ratio (PSNR) also, charts are generated based on these values.

1) Mean square error (MSE): MSE values indicated a value of null for perfect alignment between both images. For similar alignment achieved, the MSE values range nearly from $0.10 \mathrm{e} 4$ to $0.12 \mathrm{e} 8$ and for misregistration, the MSE values estimated in the range of $5.9 \mathrm{e} 6$ to $6.4 \mathrm{e} 6$ [22]. The values of this evaluation are illustrated as in Fig.6. Translation shows better results compared to affine, similarity and rigid.

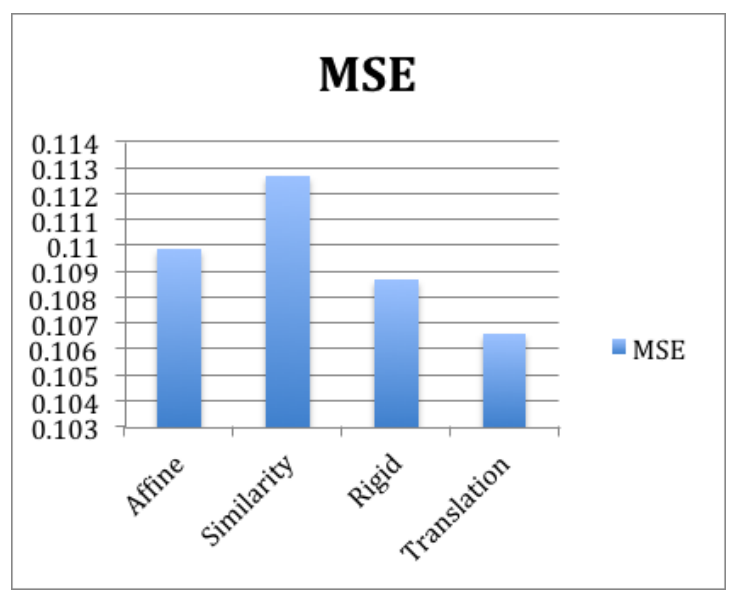

Fig. 6. Similarity Measure MSE Computed for Image Registration. 
2) Peak signal noise ratio(PSNR): PSNR is identified as a numerical measure for image registration quality based on the pixel differences between two images[26]. The Mathematical equation is provided as in equation 12 based on [26]

$\mathrm{PSNR}=10 * \log _{10}\left(\mathrm{MAX}^{2)} /(\mathrm{MSE})\right.$

Where MAX is the maximum possible pixel value of the image and MSE is mean square error between reference and target image. Fig. 7 presents image registration accuracy using PSNR to evaluate the pixel difference between these images.

A higher PSNR value indicates a higher similarity between registered images contrarily, the smaller PSNR value indicates poor similarity between images [27].From this chart, it can be seen that, translation has achieved the highest ranking among the others. Hence, signifies better accuracy found via translation transformation.

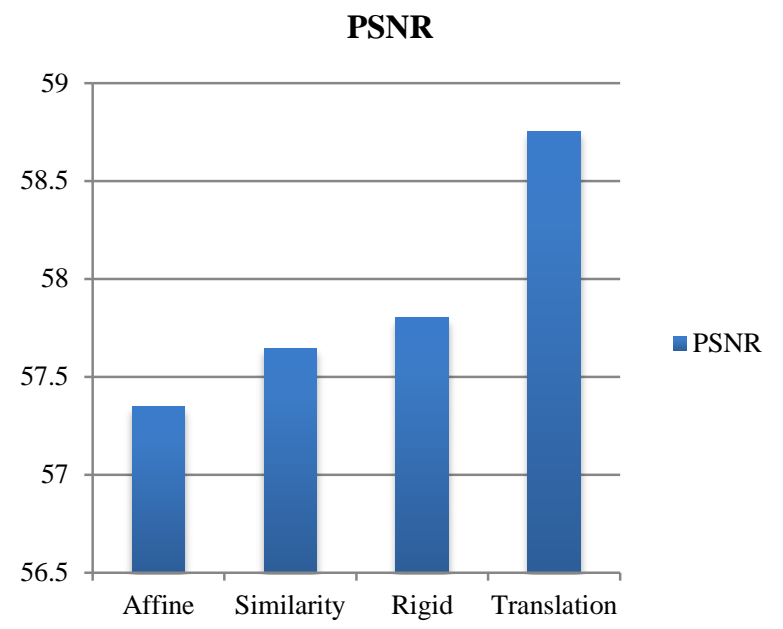

Fig. 7. Accuracy Meaurament using PSNR to Evaluate the Pixel differences between Images.

3) Result summary: Table 1 draws the registration accuracy using various measurement methods. Mean and standard deviation shows similar results for all transformation type while MSE and PSNR display dissimilar results for each transformation type.

TABLE I. SUMMARY OF LINEAR TRANSFORMATION RESULTS

\begin{tabular}{|l|l|l|l|l|}
\hline \multicolumn{5}{|l|}{ Registration Accuracy } \\
\hline Model/Measures & Affine & Similarity & Rigid & Translation \\
\hline Mean & 127.5 & 127.5 & 127.5 & 127.5 \\
\hline SD & 74.05 & 74.05 & 74.05 & 74.05 \\
\hline MSE & 0.1099 & 0.1127 & 0.1087 & 0.1066 \\
\hline PSNR & $\begin{array}{l}57.3470 \\
\mathrm{~dB}\end{array}$ & $\begin{array}{l}57.6465 \\
\mathrm{~dB}\end{array}$ & $\begin{array}{l}57.8023 \\
\mathrm{~dB}\end{array}$ & $58.7549 \mathrm{~dB}$ \\
\hline
\end{tabular}

\section{CONCLUSIONS}

Transformation types are crucial for the success of image registration. A transformation type is selected to reflect geometric differences between two images in image registration. Often, selection of the right transformation type for image registration is undeniably challenge. To make this selection as effective as possible, an experimental mechanism has to be done to determine suitability in particular image registration. Hence to understand of the above problem, a comparison between linear geometrical transformations on medical image registration is presented in this paper. The priciest alignment among all transformation types has been successfully investigated. In this study, intensity based image registration method has been used. Mean square error metric is used as similarity metric and regular step gradient descent optimizer is used as an optimization method. Data set has been acquired from Hospital Canselor Tuanku Muhriz. Based on the Mean Square Error similarity measure analysis, the translation transformation performed better in aligning the mono-modal MR images with MSE values lesser than other transformation functions. Translation control points distributed globally coverage. Thus higher similarity found between reference image and the transformed target image.

\section{ACKNOWLEDGMENT}

The authors would like to thank Dana Lonjakan Universiti Kebangsaan Malaysia (UKM) for financial support in publishing this paper, and administrations in Hospital Canselor Tuanku Muhriz and UKM for their good cooperation and helps in doing this project. An appreciation also goes to members of Medical Computing Lab for the ideas and fruit full discussions.

\section{REFERENCES}

[1] U. Vovk, F. Pernuš, and B. Likar, "A Review of Methods for Correction of Intensity Inhomogeneity in MRI," IEEE Trans. Med. Imaging, vol. 26, no. 3, 2007.

[2] G. Lematre, "Computer-Aided Diagnosis for Prostate Cancer using Multi-Parametric Magnetic Resonance Imaging,” 2016.

[3] L. Liu, Z. Tian, Z. Zhang, and B. Fei, "Computer-aided Detection of Prostate Cancer with MRI," Acad. Radiol., vol. 23, no. 8, pp. 10241046, 2016.

[4] A. Goshtasby, "Transformation functions for image registration," 2003.

[5] L. Liu, Z. Tian, Z. Zhang, and B. Fei, "Computer-aided Detection of Prostate Cancer with MRI: Technology and Applications," Acad. Radiol., vol. 23, no. 8, pp. 1024-1046, 2016.

[6] J. O. Barentsz et al., "ESUR prostate MR guidelines 2012," Eur. Radiol., vol. 22, no. 4, pp. 746-757, Apr. 2012.

[7] G. Lemaître, R. Martí, J. Freixenet, J. C. Vilanova, P. M. Walker, and F. Meriaudeau, "Computer-Aided Detection and diagnosis for prostate cancer based on mono and multi-parametric MRI: A review," Comput. Biol. Med., vol. 60, pp. 8-31, May 2015.

[8] Noha Mohamed AbdelMaboud a, $1 *$, Hytham Haroon Elsaid a, and 2 Essam Aly Aboubeih b, "The role of diffusion - Weighted MRI in evaluation of prostate cancer."

[9] E. de Kerviler, "[Imaging in prostate cancer]," Rev Prat, 2013. [Online]. Available: https://www.medscape.com/viewarticle/742986_3. [Accessed: 19-Oct-2018].

[10] T. Higaki et al., "ImagIng PhysIcs Introduction to the Technical Aspects of Computed Diffusion-weighted Imaging for Radiologists," RadioGraphics, vol. 38, pp. 1131-1144, 2018. 
[11] M. Hara et al., "A new phantom and empirical formula for apparent diffusion coefficient measurement by a 3 Tesla magnetic resonance imaging scanner," Oncol. Lett., vol. 8, no. 2, pp. 819-824, Aug. 2014.

[12] A. X-ray, "Matching in 2D," 2000.

[13] A. Goshtasby, "Transformation functions for image registration," 2003.

[14] C.-W. Wang and H.-C. Chen, "Bioimage informatics Improved image alignment method in application to X-ray images and biological images," vol. 29, no. 15, pp. 1879-1887, 2013.

[15] E. Irmak and M. Burak Türköz 2, "A Useful Implementation of Medical Image Registration for Brain Tumor Growth Investigation in a Three Dimensional Manner," IJCSNS Int. J. Comput. Sci. Netw. Secur., vol. 17, no. 6, 2017.

[16] "Intensity-based automatic image registration - MATLAB \&amp; Simulink." [Online]. Available: https://www.mathworks.com/help/images/intensity-based-automaticimage-registration.html. [Accessed: 12-Oct-2018].

[17] A. Heidenreich et al., "Guidelines on Prostate Cancer. Update," 2011.

[18] Y. Zhang, "Image processing using spatial transform," 2009 Int. Conf. Image Anal. Signal Process., vol. 2, no. 1, pp. 282-285, 2009.
[19] S. Uchida, Image processing and recognition for biological images, vol. 55, no. 4. 2013.

[20] P. Wittman, Lec 12: Image Registration. The Citadel, 2014.

[21] A. A. Goshtasby, "2-D and 3-D Image Registration," Wiley Press, 2004.

[22] R. Joshi, R. Cook, and C. It, "An Analysis of Rigid Image Alignment Computer Vision Algorithms," 2012.

[23] E. IRMAK, E. ERÇELEBİ, and A. H. ERTAŞ, "Brain tumor detection using monomodal intensity based medical image registration and MATLAB," Turkish J. Electr. Eng. Comput. Sci., vol. 24, no. January 2016, pp. 2730-2746, 2016.

[24] L. Lo Presti and M. La Cascia, "Multi-modal Medical Image Registration by Local Affine Transformations," 2018.

[25] R. Panda, S. Agrawal, M. Sahoo, and R. Nayak, "A novel evolutionary rigid body docking algorithm for medical image registration," Swarm Evol. Comput., vol. 33, pp. 108-118, 2017.

[26] Y. Tanabe and T. Ishida, "Quantification of the accuracy limits of image registration using peak signal-to-noise ratio," Radiol. Phys. Technol., vol. 10, no. 1, pp. 91-94, Mar. 2017.

[27] M. Sen Pan, J. T. Tang, and X. L. Yang, "Medical image registration based on SVD and modified PSNR," 2011. 